\title{
Percolation thresholds in temperature dependence of electric conductivity of collagen films
}

\author{
Department of Biophysics, Poznan University of Medical Sciences, Poland \\ e-mail:mgauza@ump.edu.pl
}

\begin{abstract}
Temperature measurement of electrical conductivity $\sigma$ were carried out from 395 to $543 \mathrm{~K}$. The obtained dependence indicates an increase in $\sigma$. The temperature dependence of the electrical conductivity allowed to observe the release of the free water, bound water and structural water. Studies on electric conductivity of collagen indicate the occurrence of percolation threshold, which comprises free release of water and change its properties from semiconducting to dielectric one.
\end{abstract}

Key words: electric conductivity, temperature, percolation threshold, collagen.

\section{Introduction}

The temperature dependence of electrical conductivity can be applied for the evaluation of the influence of different physical and chemical factors on such materials as: polymers, composites and biological materials, for example, collagen, bone and keratin; and materials of semiconducting and dielectric properties. The method allows to the phase change occurring in the studied material. The phase change may include subsequent changes of one or several physical quantities. Often, the important change in physical properties of the system such as concentration, electric resistance and spontaneous magnetization take place. Usually, in complex materials, all components participate in the phase transition [1].

Percolation phenomena have been observed in the heterogeneous hydrated biological materials [2]. Percolation transitions belong to the second type phase 
transitions [3]. Also studies on electric conductivity of collagen indicate the occurrence of percolation effect. The percolation is defined as the problem of determining the critical threshold concentration of conducting links in a percolation network. In the network, clusters of conducting links are formed and the lattice may transform from an insulator to a conductor $[4,5]$. Percolation characterizes the solid structure, its porosity, complexity and types of substructures open or closed, whereas permeability characterises the entire system 'solid+fluid' of collagen, and it can be compared to electrical resistance [6]. If during a phase transition, the percolation threshold is achieved, suddenly, the long-range interactions arise [7] and the transition from one system into another takes. Percolation path provides the flow of fluid or current system from one the side to the other.

Collagen is the main biopolymer in living organisms and is the main component of skin and bone $[8,9]$. The macromolecule of collagen is a complex molecule made of three polypeptide chains, which form the left-turn super helix. Untill now, bovine collagen has been used in cosmetology and implantology. The observed transmission of spongiform encephalophaties (BSE) to humans resulted in the interest in the safety of the bovine derived products. Therefore, development of new sources of collagen is recently observed. Fish skin seems to be one of such safe sources of collagen. Unfortunately, the temperature tolerance of the fish skin collagen (FSC) is limited and longer exposition to heat leads to thermal denaturation. Fish collagen is the natural protein received from some fish and has even better cosmetic specificity than mammalian collagen. Collagen isolated from fish skin by hydration seems to maintain its native structure $[10,11]$. The temperature of collagen denaturation depends on water content, cross-linking degree and hydroxyproline content. Bound water seems to be of fundamental importance for the understanding of physicochemical structural and biological properties of biopolymers, in particular proteins [12-15].

Many factors may influence the temperature dependence of collagen electric conductivity, which is sensitive to structural changes, water content and ageing processes. Electrical properties of solid-state proteins allow to classify them as conducting semiconducting or dielectric materials. The electrical conductivity of semiconductors ranges from about $10^{3}$ to $10^{-9} \Omega^{-1} \mathrm{~cm}^{-1}$. For comparison, a maximum conductivity of $10^{7}$ for good conductors and a minimum conductivity of $10^{-17} \Omega^{-1}$ $\mathrm{cm}^{-1}$ for good insulators [16]. 
The aim of the presented study was to find the percolation effect on the temperature dependence of electric conductivity of fish-skin collagen. The analysis allows to follow the release of free, bound and structural water along with thermal denaturation.

\section{Material and metod}

The collagen used in the experiment was isolated from the skin of Salmo salar, by the method described in $[10,11]$. Fish skin collagen in the gel form, containing $9 \%$ of the pure collagen was poured on the Petri dish and dried. Next, the cylindrical samples were cut. The samples were $0.08 \mathrm{~mm}$ thick, $10 \mathrm{~mm}$ in diameter and had the mass of $(40.0 \pm$ $0.2) \mathrm{mg}$. Measurements were carried out during continuous heating run. Heating was carried out for two groups of samples. Samples belonging to the first group were heated up to the temperature of $543 \mathrm{~K}$ (the first heating run), cooled to the ambient temperature, heated again to $543 \mathrm{~K}$ (the second heating run) and kept at this temperature to release the bound water. The second group of samples was heated up to $395 \mathrm{~K}$, kept at this temperature for an hour to release free water, and cooled down to the ambient temperature. Heating was repeated three times. Subsequent heating runs were stopped at: $475 \mathrm{~K}, 500 \mathrm{~K}$ and $543 \mathrm{~K}$. Each heating run was finished with cooling samples to the ambient temperature. The aim of the subsequent heating runs allowed to eliminate local maxima and flexion points observed on thermograms during first,

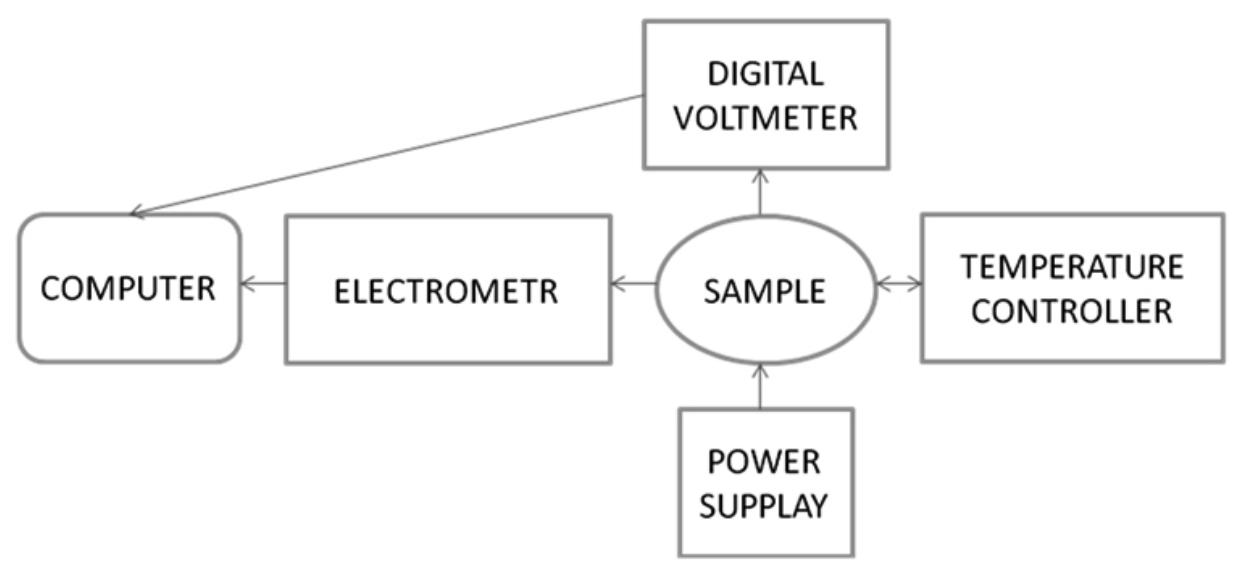

Figure 1. The block diagram of the experimental set-up. 
continuous heating. All measurements were performed in air, under atmospheric pressure. The temperature of the sample was measured independently, using constantan-copper thermocouple located in the proximity to the sample surface. The EMF of the thermocouple was indicated by a digital voltmeter. The temperature dependence of electric conductivity was measured in the temperature range 291-543K. With a constant rate of $4 \mathrm{~K} / \mathrm{min}$. provided by the programmable temperature controller. The dc voltage was applied to the sample's electrodes and generated current was measured by means of an electrometer (Keithley 6514). The block diagram of the experimental set-up is shown in Figure 1. The electrical conductivity $\sigma$ was calculated on the basis of the current, voltage and sample size, using the following equation:

$$
\sigma=\frac{d \cdot I}{S \cdot U}
$$

where $S$ is the cross-sectional surface area, $d$ is the thickness of the sample, $U$ is the voltage applied to the sample and $I$ is the measured current.

\section{Results and discussion}

The resultants obtained during the constant heating run in the temperature range 291-543K are shown in Figure 2. During the single heating two maxima were observed at the temperatures of $359 \mathrm{~K}$ and $454 \mathrm{~K}$ (Figure 2a). Most probably, the local maximum is the manifestation of the phase transition caused by temperature increase. The process is irreversible because the maximum disappeared during the second heating run (Figure $2 \mathrm{~b})$. The maximum at $354 \mathrm{~K}$ results form the release of free water, whereas the maximum at $545 \mathrm{~K}$ is related to the thermal denaturation [17]. Results obtained during subsequent heating run are shown in Figure 3 and Figure 4. During the first heating run in the temperature range of $291-395 \mathrm{~K}$, the maximum observed at the temperature of about $343 \mathrm{~K}$ was, probably, related to the release of the free water (Figure 3) [18, 19-21]. The exponential increase of conductivity observed during the second, third and fourth heating run (Figure 4) without any local maximum is caused by the more accurate water release carried out during the subsequent heating runs. The temperature of collagen denaturation depends on its humidity, whose decrease leads to the higher thermal stability. The large increase in conductivity was found in the temperatures over 390, 400 
a)

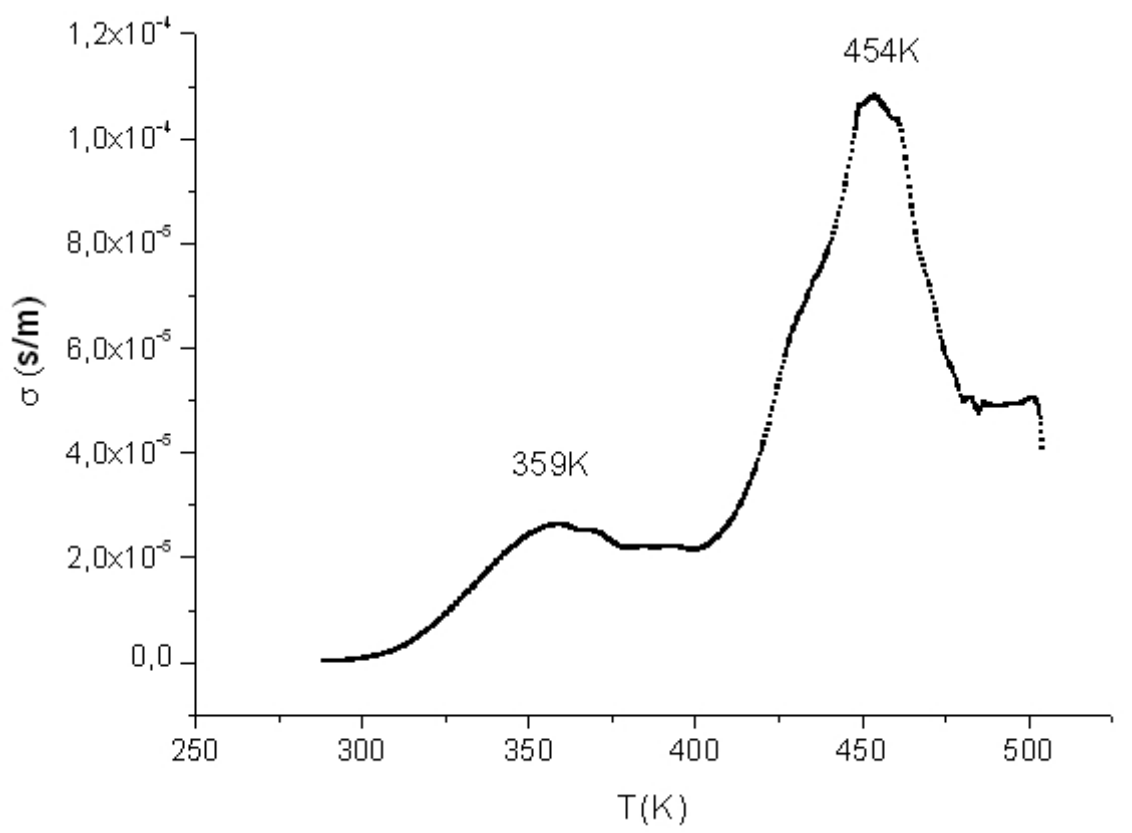

b)

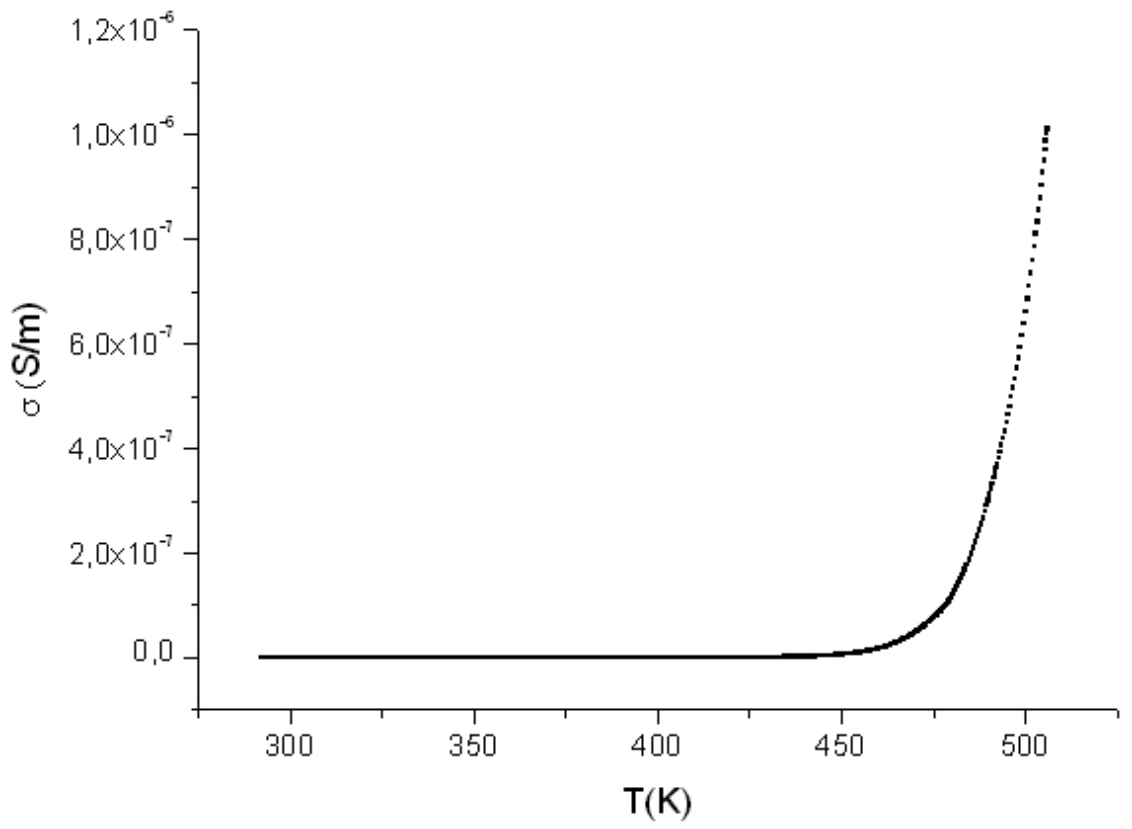

Figure 2. Temperature dependence of electrical conductivity: (a) during first heating run; (b) and second heating run. 


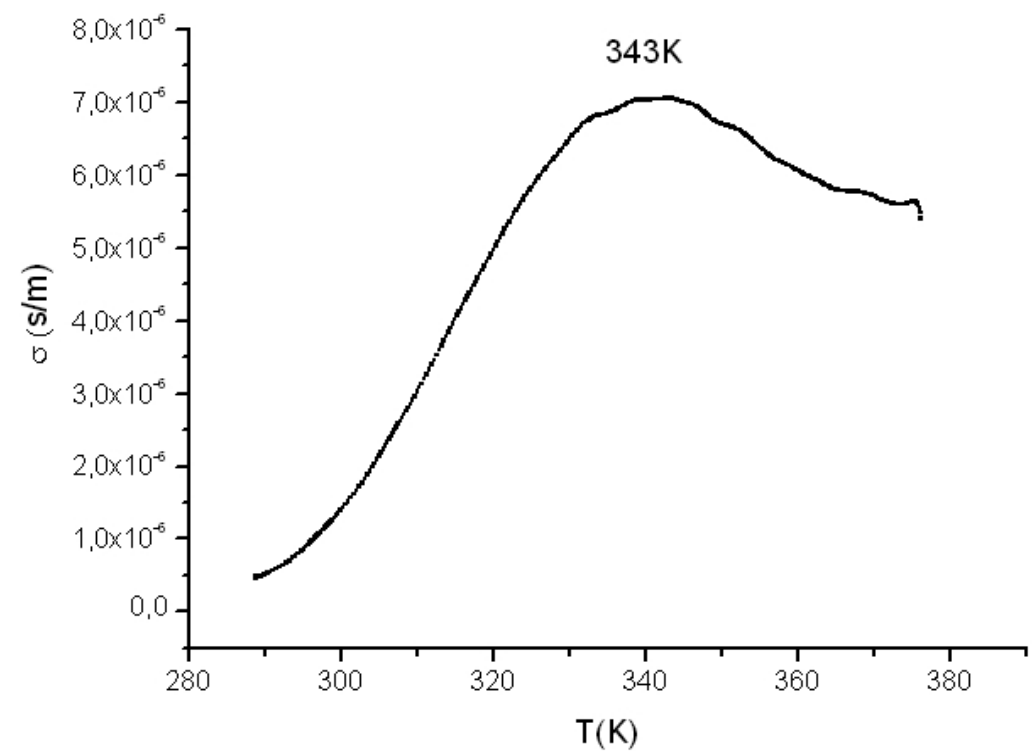

Figure 3. Temperature dependence of electrical conductivity during the first heating run.

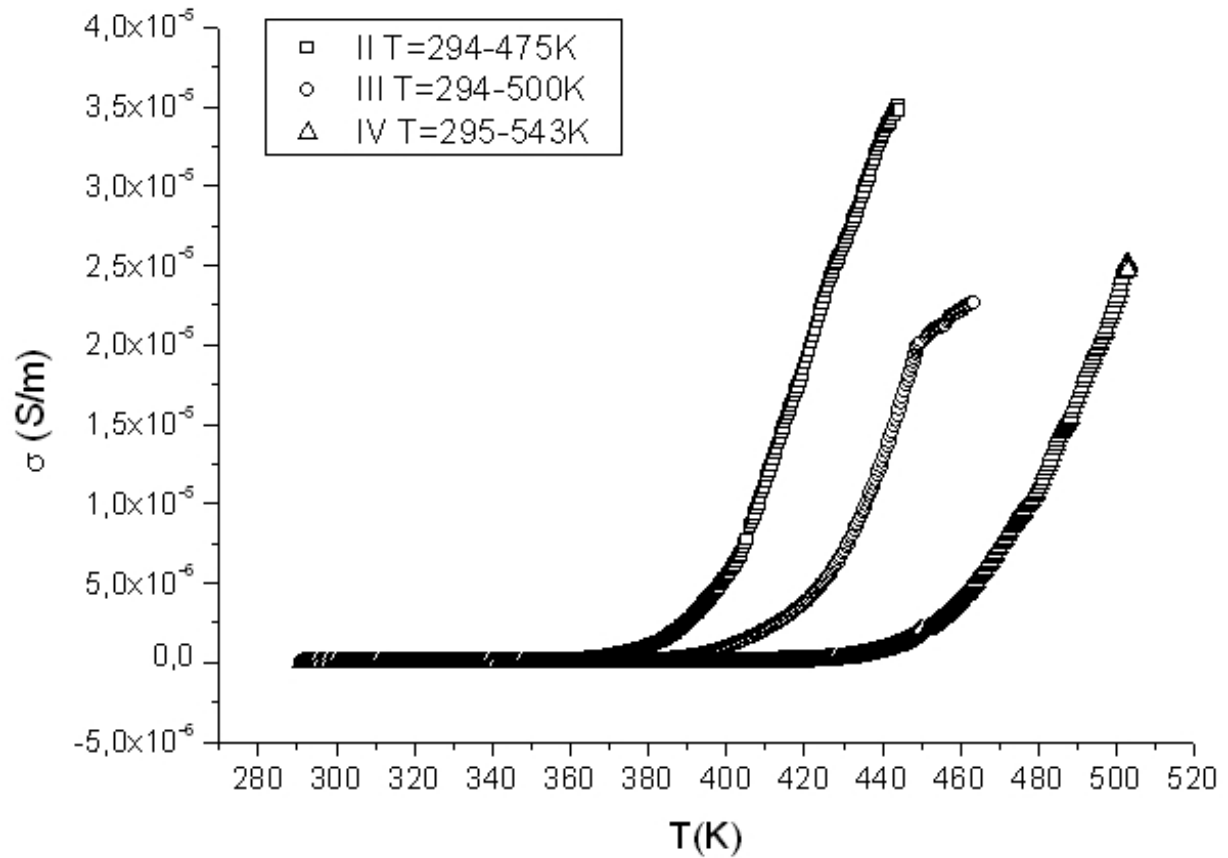

Figure 4. Temperature dependence of electrical conductivity during next heating run. 
and $450 \mathrm{~K}$ for the second, third and fourth heating run, respectively. This reflects the increasing thermal stability of the studied material caused by the reduced humidity.

Heating of natural biological materials results in their thermal degradation and structural changes. These changes affect the electric conductivity of the materials which allows to use the temperature studies of the electric conductivity to determine the temperature of the phase transition such as denaturation [22]. The electrode effects was negligible because of material conductivity, which was lower than $10^{-8} \Omega^{-1} \mathrm{~cm}^{-1}$ $[23,24]$.

\section{Conclusion}

The studied material is an example of the porous system possessing percolation thresholds [25]. Their presence was deduced on the basis of the temperature dependence of the electrical conductivity where the process of water release and thermal denaturation were manifested. The release of water, observed during heating, resulted in the decreased electric conductivity. Therefore reduction of the hydration level was assumed to be a type of the percolation threshold. If the material achieves this threshold, the subsequent heating revealed the monotonous increase in electrical conductivity. Also heating above the denaturation temperature can be considered as the percolation process during which the material is transformed irreversibly from the native state to denaturated one.

\section{Acknowledgment}

Authors are grateful to 3-Helisa, Gdynia for granting a material used in the experiment. 


\section{References}

[1] Gonczarek R, Krzyzosiak M. Critical parameters in the superconducting singular Fermi liquid model. Phys C. 2006; 445-448: 158-161.

[2] Stauffer D, Aharony A. Introduction to Percolation Theory, 2nd ed. London: Taylor and Francis; 1992.

[3] Nomura Y, Toki S, Ishii Y, Shirai K. The physicochemical property of shark type I collagen gel and membrane. J Agric Food Chem. 2000; 48(6): 2028-2032.

[4] Przybylski JE et al. Patent US 7285638 B2 2007.

[5] Sokolowska D, Krol-Otwinowska A, Moscicki JK. Water-network percolation transitions in hydrated yeast. Phys Rev E. 2004; 70: 052901.

[6] Gutfraind R, Sheintuch M. Scaling Approach to Study Diffusion and Reaction Processes on Fractal Catalysts. Chem Eng Sci. 1992; 47: 4425.

[7] Fonseca MJ, Juve A, Lorincz Z, Reig F, Alsina MA. Physicochemical Study of Hydrophobic Derivatives of Collagen Fragments. J Colloid Interface Sci. 1998; 205 : 141-148.

[8] Berg JM, Tymoczko JL, Stryer L. [Biochemistry] Warszawa: PWN; 2005. In Polish.

[9] Fratzl P. Collagen, Structure and Mechanics. New York: Springer; 2008. p. 506.

[10] Przybylski JE, Siemaszko-Przybylska K. Patent 190737; RP 2002.

[11] Romm F. Theories and theoretical models for percolation and permeability in multiphase systems: comparative analysis. Adv Colloid Interface Sci. 2002; 99(1): 1-11.

[12] Berendsen HJC. Water Structure. In: Theoretical and Experimental Biophysics. New York: Marcel Dekker Inc.; 1967. Vol 1, p. 1-76.

[13] Grigera JR. Introduction to the Biophysics of Water. Buenos Aires: Universitaria Buenos Aires; 1976.

[14] Hazlewood C. Accumulation and exclusion of ions in contractile tissue of developing animals. Ann N Y Acad Sci. 1973; 204: 416.

[15] Mascarenhas S. The electret effect in bone and biopolymers and the bound water problem. Ann N Y Acad Sci. 1974; 238: 36-52.

[16] Kittel C. [Introduction to Solid State Physics]. Warsaw: PWN; 2001. in Polish.

[17] Parker SP. McGraw-Hill Dictionary of Scientific and Technical Terms. 5th ed. McGraw-Hill; 1994.

[18] Hallet J. Kinetics of water solvent interaction. (Relevance of freezing to biological processes). Feder Proc Fed Am Soc Exp Biol. 1965; 24: 34-37.

[19] Kuntz ID. Hydration of macromolecules. III. Hydration of polypeptides. J Am Chem Soc. 1971; 93: 514-516.

[20] Kuntz ID, Brassfield TS. Hydration of macromolecules. II. Effect of area on protein hydration. Archs Biochem Biophys. 1971; 142: 660-664. 
[21] Kuntz ID, Brassfield TS, Law GD, Purcell GV. Hydration of macromolecules. Science. 1969; 163: 1329-1331.

[22] Kubisz L, Mielcarek S, Jaroszyk F. Changes In thermal and electric al properties of bone as result of 1 MGy - dose $\gamma$-irradiation, Int J Biol Macromol. 2003; 33: 89-93.

[23] Davies M, Hill NE, Vaugham WE, Price AH. Practical correction for electrode polarization effects. In: Dielectric Properties and Molecular Behaviour. London: Van Nostrand-Reinhold; 1969. pp. 285-287.

[24] Marzec E, Warchol W. Dielectric properties of a protein-water system in selected animal tissues. Bioelectrochem. 2005; 65: 89-94.

[25] Hansen A, Hinrichsen EL. Some remarks on percolation. Phys Scripta. 1992; 44: 55-61. 